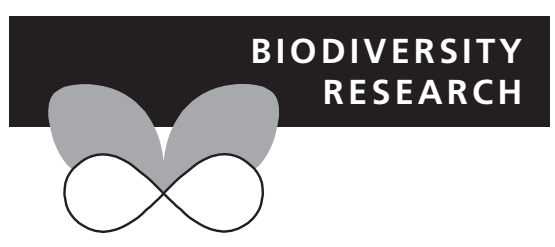

\title{
Spatially-explicit sensitivity analysis for conservation management: exploring the influence of decisions in invasive alien plant management
}

\author{
Núria Roura-Pascual ${ }^{1,2 \star}$, Rainer M. Krug ${ }^{1}$, David M. Richardson ${ }^{1}$ and \\ Cang Hui ${ }^{1}$
}

${ }^{1}$ Centre for Invasion Biology, Department of Botany \& Zoology, Stellenbosch University, Private Bag X1, Matieland 7602, South Africa, ${ }^{2}$ Àrea de Biodiversitat, Centre Tecnològic Forestal de Catalunya, Ctra. St. Llorenç de Morunys km 2, 25280 Solsona, Catalonia, Spain

${ }^{*}$ Correspondence: Núria Roura-Pascual, Àrea de Biodiversitat, Centre Tecnològic Forestal de Catalunya, Ctra. St. Llorenç de Morunys km 2, 25280 Solsona, Catalonia, Spain.

E-mail: nrourapascual@gmail.com

\begin{abstract}
Aim Decision-support models have considerable potential for guiding management strategies when problems are complex. The robustness of such decision-making processes is rarely evaluated, and the influence of decision criteria (or factors) in management decisions is seldom considered. We present a framework for a spatially-explicit sensitivity analysis by using a scheme developed to provide objective guidelines, in the form of static priority maps, for managing woody invasive alien plants (IAPs).
\end{abstract}

Location The Cape Floristic Region, South Africa.

Methods The model included seven factors related to management history, fire risk, and the age, identity, density and spread of IAPs. Each factor had a weight associated that reflected its relative importance in prioritizing areas for clearing. We changed these factor weights using three approaches of sensitivity analysis and assessed the effect of these changes on the spatial structure of the resulting priority maps in three different management regions.

Results Different outcomes arose depending on the importance given to different factors. Priority maps were most sensitive to the fire-related factors, suggesting that fire is both a crucial driver of invasion in fynbos and an overriding determinant of management options. The factor 'area burnt recently' provided crucial information for the effective clearing of IAPs. The sensitivity of the model to changes in other factors was more context specific: levels of sensitivity were highly dependent on different features of the landscape, especially the spatial heterogeneity of particular factors.

Main conclusions By clarifying the importance of factors in shaping priority maps, the sensitivity analysis framework enabled us to identify the necessary factors to produce outcomes matching a pre-selected management strategy. This is important for cost-efficient management, as acquisition and curation of data is expensive. This spatially-explicit sensitivity analysis is, thus, recommended to evaluate the robustness and generality of selected management strategies, and validate the assumptions derived from decision-making protocols.

\section{Keywords}

Biodiversity conservation planning, biological invasions, Cape Floristic Region, multi-criteria decision model, South Africa, spatially-explicit sensitivity analysis.

\section{INTRODUCTION}

Theoretical and analytical advances in conservation biogeography have greatly improved our knowledge of the spatial dynamics of taxa and assemblages, as well as the relationship between nature and humans (Whittaker et al., 2005). This improved understanding of the mechanisms behind the distribution of organisms, and the increasing complexity of the tasks 
facing managers when attempting to accommodate the multiple linkages between natural and human environments has driven the development and application of formal tools to assist the decision making for environmental management.

Multi-criteria decision models (MCDMs) are often applied to guide management when the problems at hand are complex and outcomes depend on multiple factors (de Steiguer et al., 2003), analogues to problems that confront most conservation managers. MCDMs combine social, economic and environmental factors or criteria into a manageable number of factors to identify and quantify the severity of a problem, and select a subset of optimal management strategies as the outcome. To ensure the consensus and utility of the outcomes in setting spatially-explicit priorities for management, decision models are normally developed in a participatory process involving various stakeholders, policy groups and researchers. Such decision-support tools have been applied for many purposes, e.g. identifying responses of systems to perturbations (Fürstenau et al., 2007), delimiting conservation areas (Moffett et al., 2006; Regan et al., 2007) and setting control priorities for biological invasions (Roura-Pascual et al., 2009).

Sources and levels of uncertainty in MCDMs need to be understood to attach levels of confidence to decisions (Ascough et al., 2008). One key source of uncertainty in MCDMs relates to the structure of the model; uncertainty arises from the use of inappropriate factors and expressions in representing real-world system (Harwood \& Stokes, 2003). Thus, the robustness of the decision-making process and the influence of different factors in determining results (i.e. decisions) must be evaluated to allow for management to improve over time (Hyde et al., 2005; Fuller et al., 2008). Such steps are seldom taken in conservation management (Moffett et al., 2006; Valente \& Vettorazzi, 2008). Consequently, applied decision-support systems have little heuristic value and fundamental decision questions need to be addressed afresh at each iteration. Evaluating the sensitivity of decision models paves the way for considering alternative strategies (Neubert \& Caswell, 2000; Buckley et al., 2005). An important task for conservation biogeographers is to explore the sensitivity of management outcomes to various model effects and the best ways in which different societal objectives can be incorporated in modelling frameworks (Whittaker et al., 2005).

In this study, we provide a framework for a spatially-explicit analysis that evaluates the sensitivity of model-based management prescriptions to changes in the relative importance assigned to different decision criteria (here referred as factors). As an example, we apply the framework to a decision model developed to prioritize areas for invasive plant control to three study regions in South Africa's Cape Floristic Region (RouraPascual et al., 2009). Based on the spatial consequences of changing the relevance of factors included in the decision model, the sensitivity analysis aims to gain a better understanding of: (1) the role of different factors in determining the decision outcomes (i.e. static priority maps that delineate priorities across management regions); (2) the minimum data required to generate results that match the original decision model (defined by experts in a participatory workshop), without losing relevant information; (3) the divergences among the original decision model and other management strategies currently under way; and (4) the sensitivity of the decision model to the particularities of different geographic regions. Answers to these questions enable us to identify the main sources of uncertainty associated with our decision model and assist managers in setting up management actions that consider the influence of factors included in the analysis.

\section{METHODS}

\section{Prioritization of areas for alien control: the decision model case}

The sensitivity analysis was applied to a MCDM aimed at identifying areas for the control of the most important woody invasive alien plants (IAPs) at the landscape scale in South Africa's Cape Floristic Region (regional or national-scale planning typically demands the use of other factors that are not addressed here). The MCDM was developed through a participatory process with managers and researchers experts on various aspects of IAPs. The participatory process adopted the structured Analytical Hierarchy Process to determine the relative importance of factors at each level of the model hierarchy (Saaty, 1980). Details on the development and structure of the MCDM are given elsewhere (Roura-Pascual et al., 2009). For simplicity, here we only present the core of our decision model - the stand-attributes module - which sets the priorities for the management of invaded stands in a distinct, geographically defined, management region within the Cape Floristic Region.

This module stand-attributes (hereafter referred as decision model) includes seven factors related to management history, fire risk, and the identity, age, density and spread of IAPs: 'area burnt recently' (ABR), 'density of IAPs' (DoI), 'spread based on topography' (SbT), 'areas at fire risk' (AFR), 'age of IAPs' (AoI), 'identity of IAPs' (IoI) and 'last clearing operation' (LCO). These factors were further divided into sub-factors (see Table 1 for more details). The relative importance (or weight) of each factor and sub-factor was assigned during the development of the MCDM (Roura-Pascual et al., 2009). The sum of all factors' weight equals one, as well as the sum of all sub-factors' weight within each particular factor. For example, the factor 'spread based on topography' has an importance of 0.17 compared to the rest of the factors, and its sub-factors 'planar and pit', 'channel' and 'pass, ridge and peak' have a sub-weight of 0.07, 0.28 and 0.65 , respectively. Overall, factors related to the fire-prone nature of the ecosystem and the characteristics of the invasive stands emerged as the pivotal features for setting spatiallyexplicit priorities for management (Table 1).

To derive a spatial representation of the decision model for a given management region, the weights of factors and subfactors were converted into a map indicating prioritized areas for IAPs control within a GIS interface. The region was divided into cells of equal resolution $(100 \mathrm{~m} \times 100 \mathrm{~m})$ and each cell 
Table 1 Factors and sub-factors included in the stand-attributes module of the multi-criteria decision model developed by RouraPascual et al. (2009) for setting spatially-explicit priorities for management of invasive alien plants (IAPs) at local scales in the Cape Floristic Region. Weights associated with each factor $\left(\omega_{i}\right)$ indicate their importance (0-1) within the module and all values sum up to 1 . Weights for each sub-factor $\left(v_{i j}\right)$ indicate their relative importance (0-1) within that specific factor and sum up to 1 . For example, the factor relating to the density of IAPs has an importance of 0.17 compared to the rest of the factors within the stand-attributes module and the sub-factor 'occasional' an importance of 0.39 within that factor.

\begin{tabular}{|c|c|c|c|}
\hline Factors and sub-factors & $\omega_{i}$ & $v_{i j}$ & Description \\
\hline $\begin{array}{l}\text { Are burnt recently } \\
\qquad \begin{array}{l}\text { Yes } \\
\text { No }\end{array}\end{array}$ & 0.40 & $\begin{array}{l}0.90 \\
0.10\end{array}$ & $\begin{array}{l}\text { Areas burnt between } 2002 \\
\text { and } 2004 \text { where the } \\
\text { emerging vegetation is easy } \\
\text { to visualize and remove at } \\
\text { low cost }\end{array}$ \\
\hline $\begin{array}{l}\text { Density of IAPS } \\
\text { Close } \\
\text { Dense } \\
\text { Medium } \\
\text { Scattered } \\
\text { Very scattered } \\
\text { Occasional } \\
\text { Rare }\end{array}$ & 0.17 & $\begin{array}{l}0.03 \\
0.04 \\
0.06 \\
0.10 \\
0.20 \\
0.38 \\
0.19\end{array}$ & $\begin{array}{l}\text { Density of IAPs within each } \\
\text { stand according to } \\
\text { Working for Water } \\
\text { Program (2003) }\end{array}$ \\
\hline $\begin{array}{l}\text { Spread based on } \\
\text { topography } \\
\text { Planar and pit } \\
\text { Channel } \\
\text { Pass, ridge } \\
\text { and peak }\end{array}$ & 0.17 & $\begin{array}{l}0.07 \\
0.28 \\
0.65\end{array}$ & $\begin{array}{l}\text { Potential for dispersal of } \\
\text { invaded populations based } \\
\text { on their current topographic } \\
\text { position. The most rapid } \\
\text { spread is likely to occur on } \\
\text { exposed sites upwind or } \\
\text { close to water courses }\end{array}$ \\
\hline $\begin{array}{l}\text { Area at fire risk } \\
\text { Low } \\
\text { Medium } \\
\text { High }\end{array}$ & 0.13 & $\begin{array}{l}0.07 \\
0.28 \\
0.65\end{array}$ & $\begin{array}{l}\text { Probability of a fire occurring } \\
\text { in a stand, based on } \\
\text { previous fires and position } \\
\text { in the landscape }\end{array}$ \\
\hline $\begin{array}{l}\text { Age of IAPs } \\
\text { Adult } \\
\text { Sapling } \\
\text { Seedling }\end{array}$ & 0.06 & $\begin{array}{l}0.11 \\
0.64 \\
0.26\end{array}$ & $\begin{array}{l}\text { Life stage of the IAPs, which } \\
\text { reflects the age of } \\
\text { reproductive maturity and } \\
\text { the visibility of the plants, } \\
\text { according to Working for } \\
\text { Water Program (2003) }\end{array}$ \\
\hline $\begin{array}{l}\text { Identity of IAPs } \\
\text { Hakea } \\
\text { Acacia } \\
\text { Pinus }\end{array}$ & 0.05 & $\begin{array}{l}0.10 \\
0.26 \\
0.64\end{array}$ & $\begin{array}{l}\text { Species of the genera Acacia, } \\
\text { Hakea and Pinus object of } \\
\text { clearing operations, ranked } \\
\text { according to its invasive } \\
\text { potential }\end{array}$ \\
\hline $\begin{array}{l}\text { Last clearing operation } \\
\text { No treatment } \\
\text { Initial } \\
\text { Follow-up } \\
\text { Maintenance }\end{array}$ & 0.03 & $\begin{array}{l}0.05 \\
0.30 \\
0.55 \\
0.10\end{array}$ & $\begin{array}{l}\text { Stage of the last clearing } \\
\text { operations conducted in the } \\
\text { stand according to } \\
\text { Working for Water } \\
\text { Program (2003) }\end{array}$ \\
\hline
\end{tabular}

was characterized by seven attributes, one for each factor included in the stand-attributes module. Cell attributes were extracted from various digital coverages (e.g. time since the last fire, distribution and density of alien species, topography, history of the clearing operations) that were available for the respective region. A priority value was then calculated for each cell based on cell properties and the weights assigned to the different factors included in the stand-attributes module. This resulted in a priority map for the selected region, which is a spatial representation of the decision model and constitutes the end product on which managers can base their control strategies. The priority map generated using the factor and sub-factor weights of the original decision model selected by expert agreement (Table 1) will be referred to as 'consensus prioritization' hereafter. A more detailed explanation of the conversion of the weights of factors and sub-factors to produce a priority map is given in Appendix S1.

\section{Sensitivity analysis framework}

To examine the full range of variation in the original set of factor weights and consider other potential alternatives to the original decision model (Table 1), we altered the relative importance of factors using three different sensitivity approaches (one-dimensional weights, random weights and selected weights) that differ in the way that the factor weights are modified. The weights assigned to sub-factors were kept unchanged, except for the selected-weights approach where several specific strategies were evaluated. The effect of altering the weights assigned to the factors on the distribution of the priorities in a given region was then evaluated in a spatial dimension, after converting each set of new weights into a priority map and comparing it with the map derived from the original decision model (i.e. the consensus prioritization).

To assess the robustness of the decision model across geographic regions, we ran the sensitivity analysis in three management regions within the Cape Floristic Region: the Cape Peninsula; the Agulhas Plain (including the De Hoop Nature Reserve); and the Outeniqua, Wilderness and Knysna regions. These management regions were selected for comparison because they have distinct sets of biophysical and socioenvironmental conditions that diversity opportunities for management and therefore the state and dynamics of invasions (see Roura-Pascual et al., 2009). While woody IAPs on the Cape Peninsula are relatively under control, the extent and density of invasive populations in the other regions are increasing.

Each priority map derived from the sensitivity analysis was compared with the consensus prioritization using two dissimilarity and information indices: Earth Mover's Distance (EMD) and Shannon diversity. The EMD, also called Mallows Distance (Levina \& Bickel, 2001), is a mathematical measure for evaluating dissimilarity between two distributions. Distributions are interpreted as different ways of piling up a certain amount of earth, and the EMD index is calculated as the minimum cost of turning one pile into the other, taking into 
account the distances between the different features (Ling \& Okada, 2007; Krug \& Eddelbuettel, 2009). The EMD was originally conceived as a distance measure to evaluate the differences between two images (Peleg et al., 1989), and it is used in image retrieval and histogram comparison (Rubner et al., 2000; Ling \& Okada, 2007). The Shannon diversity index $(\mathrm{H})$ is a synthetic index that quantifies the amount of information contained in an event (Shannon, 1948). It is not an absolute quantity, but measures diversity in categorical data considering both the number and abundance of the categories (Anderson, 2008). Besides its numerous applications in information theory, the Shannon index is a well-established measure of biological diversity (Hurlbert, 1971).

To our knowledge, this study is the first application of EMD and $\mathrm{H}$ indices for quantifying differences in management priorities. To render the priority maps comparable, we first standardized the values at the cell level so that the sum of all cells for a given region equals one. We were not interested in the absolute value of cells, but on the relative value in relation to other cells. The EMD was thus used to compare the priority maps derived from the sensitivity analysis with the consensus prioritization, and the $\mathrm{H}$ index to capture the multidimensional information contained in each priority map. They were simply used as relative distance metrics; for example, EMD is only able to identify whether a priority map A is more different from the consensus prioritization than a priority map B in a given region, but not to explain why it happens. The underlying causes producing these differences need to be interpreted a posteriori by visual comparison. Refer to Fig. S3 for an example of the behaviour of the EMD and $\mathrm{H}$ indices in an hypothetical region. All the analyses were performed in R ( $\mathrm{R}$ Development Core Team, 2008), and the GIS manipulations performed in GRASS GIS software v. 6.2.3 (GRASS Development Team, 2007).

\section{Approach 1: one-dimensional weights}

This sensitivity approach evaluates the effect of changing the weight assigned to a single factor by increasing it from 0 to 1 in steps of 0.01 while holding constant the ratios among the weights assigned to the other factors, so that all weights add up to 1 (Moffett et al., 2006). This resulted in $7 \times(1 / 0.01)=700$ new sets of weights, which were then converted into priority maps and evaluated as described above. EMD values comparing the dissimilarity between each priority map and the consensus prioritization, as well as the level of information contained in each map as measured by the Shannon diversity index, were graphed to evaluate the effect of altering the weight of each factor separately. This one-dimensional weights approach allows to evaluate the relative influence of each factor separately but ignores interactions that result from modifying simultaneously the weights of multiple factors (Butler et al., 1997).

\section{Approach 2: random weights}

To explore the entire spectrum of possible weight combinations, this approach generates weights for all factors at random while maintaining the sum of all weights equal to 1 . A total of 50,000 sets of random weights were generated. These new sets of weights were converted into priority maps and compared with the consensus prioritization by means of the EMD; the level of information associated to each map was also measured using the Shannon diversity index. The resultant EMD and $\mathrm{H}$ values were graphed to evaluate the influence of each factor. Unlike the one-dimensional weights, this approach allowed us to evaluate multiple factors simultaneously (Butler et al., 1997).

In addition to the visualization of the results, we used these 50,000 sets of randomly generated weights to conduct two additional analyses. In the first analysis, we retained two different subsets of random weights: (1) one subset containing the $1 \%$ of sets of random weights presenting the lowest EMD values (i.e. 500 sets presenting the most similar priority maps to the consensus prioritization), and (2) another subset containing the $1 \%$ subset of sets of random weights giving the highest $\mathrm{H}$ values (i.e. 500 sets presenting the most informative priority maps). For each subset separately, we compared the values assigned to each factor with the weights of the original decision model (Table 1) by means of a box-plot graph.

In contrast to the previous analysis where a subset of sets of random weights were selected, the second analysis used a model-selection procedure based on the Akaike Information Criterion (AIC) method (Akaike, 1974) to identify: (1) the set of random weights giving the most similar priority map to the consensus prioritization but with a minimum number of factors, and (2) the set of random weights giving the most informative priority map with a minimum number of factors. The AIC for a given set of random weights was a function of its maximized log-likelihood $\left(\ell_{i}\right)$ and the number of factors $\left(n_{i}\right)$ considered in that specific set of random weights:

$\mathrm{AIC}_{i}=2 n_{i}-2 \ell_{i}$

where the log-likelihood for the EMD was calculated as $\ell_{i}=\ln \left(1-\left(\mathrm{EMD}_{i} / \mathrm{EMD}_{\max }\right)\right)$, and for the $\mathrm{H}$ index as $\ell_{i}=\ln \left(\mathrm{H}_{\mathrm{i}} / \mathrm{H}_{\max }\right) . \mathrm{EMD}_{i}$ and $\mathrm{H}_{\mathrm{i}}$ correspond to the value of the EMD and $\mathrm{H}$ indices derived from a specific set of random weights, and $\mathrm{EMD}_{\max }$ and $\mathrm{H}_{\max }$ are the highest values for EMD or $\mathrm{H}$ indices in the entire 50,000 sets of random weights. Factors with a weight lower than 0.05 were considered as being 0 and consequently not included in $n_{i}$. Upon completion of this process, from all set of random weights, we chose the one presenting the lower AIC value with the fewest possible number of factors and calculated the difference in AIC with the consensus prioritization ( $\triangle \mathrm{AIC}$ ) to examine their divergences. As a rough rule of thumb, Burnham \& Anderson (2002) propose that models for which $\Delta \mathrm{AIC} \leq 2$ receive substantial support and are considered when making inferences, models having $2 \leq \Delta \mathrm{AIC} \leq 7$ have considerably less support, and models having $\triangle \mathrm{AIC} \geq 10$ receive no support. 
Approach 3: selected weights

The two sensitivity approaches described earlier enabled us to explore ways in which variations in the factor weights influence the distribution of the priorities, by revealing the relationships between the factors considered in the analyses. The selectedweights approach, in contrast, compares the original decision model against alternative strategies to understand the implications of adopting particular decisions. The conversion of these alternative strategies into a set of weights that encapsulate the mindsets that underpin most management actions against IAPs currently under way in the Cape Floristic Region was carried out using the Analytical Hierarchy Process, as we did in developing the original decision model in Roura-Pascual et al. (2009). In this case, however, we only made alterations to the weights assigned to the factors or sub-factors; no changes to the structure of the model hierarchy were allowed.

We generated three sets of selected weights: (1) one set was orientated towards increasing water production, with priority given to riparian areas in lower parts of the catchment invaded by dense stands of Acacia species (named 'Water production'); (2) a second set prioritizing areas based on previous clearing operations, giving higher priority to areas with an initial clearing operation or in follow-ups ('Maintain follow-ups'); and (3) a final set that is a slight variation of the original decision model, where management operations do not rely on fire opportunities to clear invaded stands and the potential of a stand to be a major source of invasion is minimized ('Keep clean') (Fig. 1 and Table S2). These sets of selected weights were converted into priority maps and compared to the consensus prioritizations using EMD and Shannon diversity indices.

\section{RESULTS}

\section{Approach 1: one-dimensional weights}

By systematically changing the weight assigned to each factor, we found differences between factors and geographic regions (Fig. 2). Increasing the weight assigned to the factor 'area burnt recently' produced remarkable changes in the priority maps. 'Density of IAPs' was the second factor that produced major changes in the Cape Peninsula and Agulhas Plain when its weight was above 0.9 , but not in Outeniqua where the 'last clearing operations' was the most relevant factor irrespectively of its weight. It is important to note, however, that increasing the weights assigned to the majority of factors produced a gradual and relatively small modification of the priority maps; only the factor 'area burnt recently' produced the most remarkable effects (Fig. 2a).

When examining the effects on the level of information contained in the final priority maps as measured by the Shannon diversity index, changes were only evident when factors were weighted above 0.8 (Fig. 2b). At these high weights, the level of information of the overall spatial representation dropped considerably because the influence of the factor was overwhelming and masked the roles of other factors. 'Density of IAPs' was

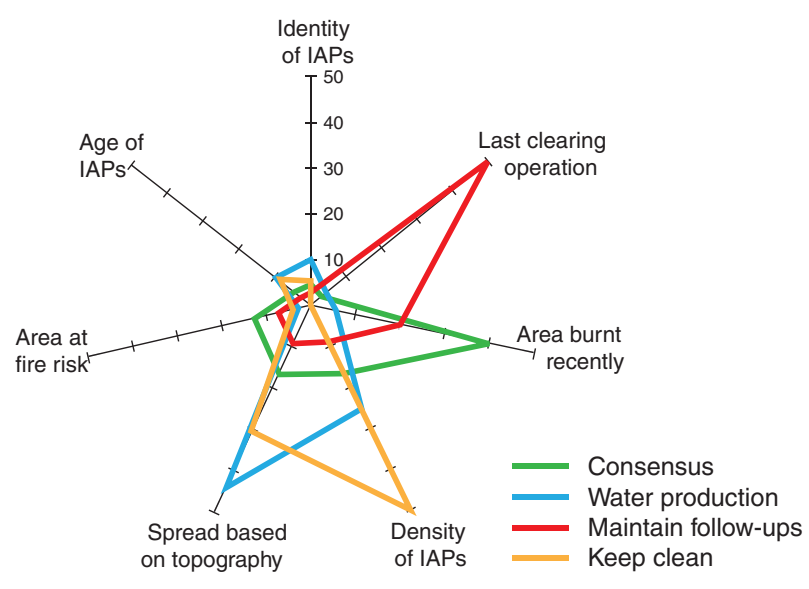

Figure 1 Visualization of strategies considered in the selectedweights sensitivity analysis in a star plot (see Table S2 for the weights assigned to the factors and sub-factors). Strategies are described according to seven axes, each one corresponding to one of the factors included in the original decision model. These axes create a seven-dimensional space, where weights assigned to each factor range from 0 (centre) to 0.5 (periphery). The total weights considered in each strategy add up to 1 .

the factor that provided the highest levels of information $(>0.8$ in all three management regions), followed by the factor 'identity of IAPs' in Cape Peninsula and Agulhas Plain.

\section{Approach 2: random weights}

When random weights were assigned to the factors, the effects on the EMD and Shannon diversity indices considering each factor separately followed a similar pattern to the one observed in the one-dimensional weights analysis (Fig. S4). The distribution of EMD values was similar across regions: the averaged EMD values increased at high factor weights, but the dispersion declined (Fig. S4a). This was expected considering that the higher the weight assigned to the factor, the lower the influence of the other factors. However, this effect was not very accentuated with the factor 'area burnt recently' (especially in Agulhas Plain) and, to a lesser extent, the 'density of IAPs'. These factors do not seem to constrain the priority maps as much as the other factors, which tend to override the influence of the rest of the factors when high weights are assigned to them. When looking at the dispersion pattern of the Shannon diversity values considering each factor separately, we found similar patterns across factors and regions: the level of information decreased considerably at high factor weights (> 0.8 approximately; Fig. S4b).

If we look closely at the $1 \%$ subset of weight combinations with the lowest EMD values (Fig. S5), the weights assigned to factors tend to be close to the values of the original decision model (Figs 3a \& S6). This result was expected, because slight variations in the original set of weights of the decision model were expected to produce similar priority maps to the consensus prioritization. However, it is important to note that the weights assigned to the factor 'area burnt recently' in Agulhas Plain, and Cape Peninsula to a lesser extent, tend to be considerably smaller 

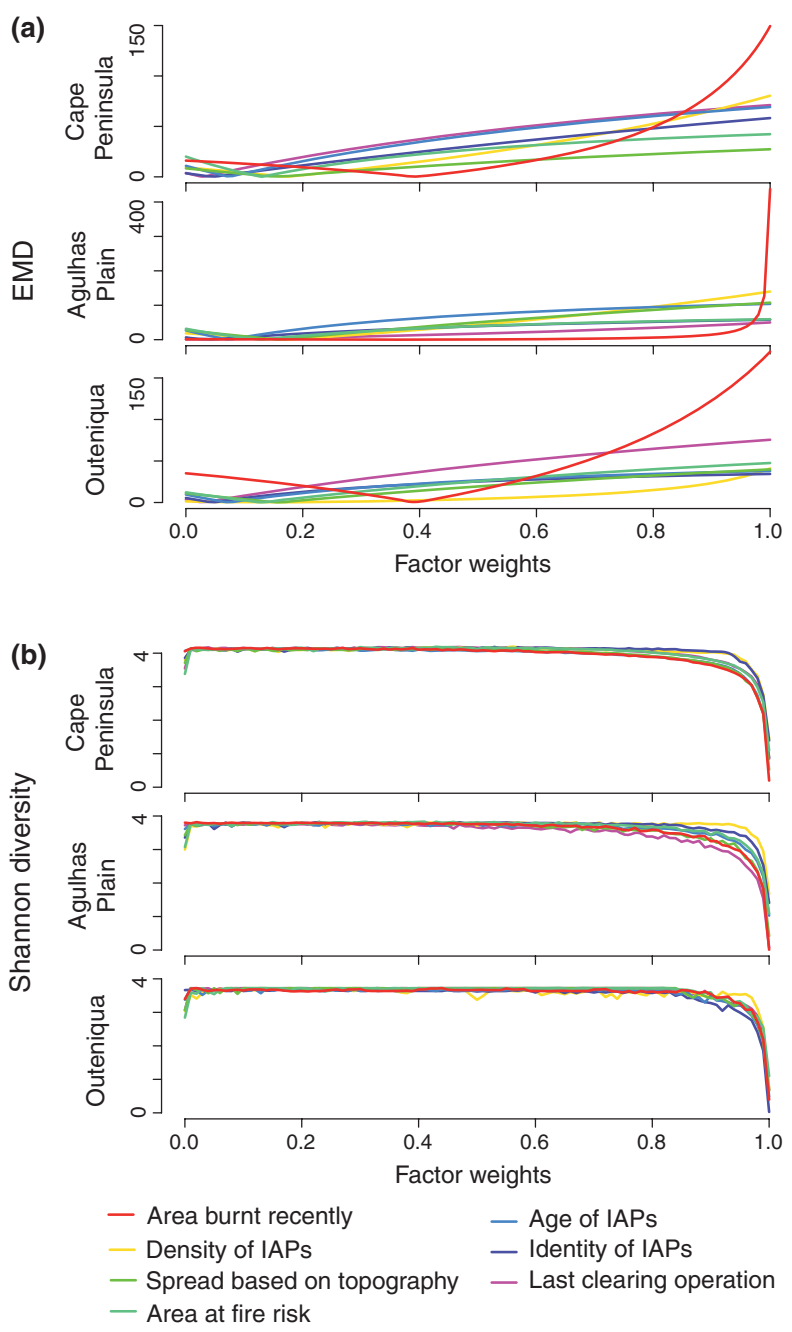

Figure 2 One-dimensional weights sensitivity analysis for three regions in the Cape Floristic Region (Cape Peninsula, Agulhas Plain and Outeniqua). The analysis was performed by changing systematically the weights assigned to each factor included in the original decision model, while maintaining the rest constant. The $x$-axis indicates the weights assigned to factors, while the $y$-axis divergences in: (a) the spatial distribution of the priorities between that specific set of factor weights and the consensus prioritization as measured by the Earth Movers Distance (EMD), and (b) the level of information as measured by the Shannon diversity index. Colours correspond to different factors. Note that graphs in (a) display different scales in the $y$-axis.

than the consensus one. These differences are very interesting, because they indicate that the consensus prioritization only represents an 'unstable' solution for yielding minimum EMD. Under these circumstances the most stable and robust set of weights should be around the median values, which are the system's attractors. To achieve the same control priorities than the decision model, we would therefore recommend the median weights, not the original weights of the decision model. This is because slight changes in the original weights produce large changes in the priority maps, whereas small mutations in the median weights do not affect the priorities for the IAPs control.
In terms of the $1 \%$ subset of sets of random weights presenting the highest Shannon diversity index (Fig. S5), the median values derived from this subset of weight combinations for the majority of the factors tend to be similar to the original weights (Figs 3b \& S6). Factors with smaller median values than the ones assigned to the original decision model are: 'areas burnt recently', 'spread based on topography' in Cape Peninsula and Agulhas Plain, and 'density of IAPs' in Outeniqua. These mismatches suggest that the decision model does not use the input data with maximum efficiency, i.e. the information contained in the input data has not been fully incorporated into the original decision model for producing the priority map (the consensus prioritization).

The protocol used to select the weight combination generating the most similar priority map to the consensus prioritization revealed that the exclusion of two or more factors produced remarkable changes in the distribution of the priorities $(\triangle \mathrm{AIC}>2$, Table $2 \mathrm{a})$. When maintaining all factors $(n=7)$, the relative importance of factors was quite similar to the original decision model in Cape Peninsula and Outeniqua, but not in Agulhas Plain where the 'density of IAPs', 'spread based on topography' and 'area at fire risk' were the most important factors. With the exclusion of one factor from the model, even though the resultant priority maps were quite similar to the consensus prioritization $(\Delta \mathrm{AIC} \leq 2$, Table $2 \mathrm{a})$, the model performed differently in the three study regions and confirmed that decision models need to be adapted to the particularities of each management region.

A similar pattern was observed when identifying the most informative combination of factor weights: the exclusion of two or more factors produced remarkable changes in the distribution of the priorities compared to the consensus prioritization $(\triangle \mathrm{AIC}>2$, Table $2 \mathrm{~b})$. For Outeniqua, it is important to note that the exclusion of one or more factors did not always alter the level of information contained in the resultant priority maps; several weight combinations achieved the same maximum value for the Shannon diversity index $(\mathrm{H}=3.72)$. In general, the relevance of factors in determining the most informative strategies differed between regions: 'density of IAPs' appeared as the most relevant in Cape Peninsula and Outeniqua, followed by the factor 'area at fire risk' to a lesser extent; contrarily, in Agulhas Plain, the most informative factors are species-specific factors such as 'age of IAPs' and 'identity of IAPs' (Table $2 \mathrm{~b}$ ). These results are in concordance with the results derived from the $1 \%$ subset of sets of random weights presenting the highest Shannon diversity index (Fig. 3b).

\section{Approach 3: selected weights}

Comparing the spatial distribution of the priorities derived from the original decision model with the three sets of selected weights (Fig. 4) by means of EMD values indicated that: (1) the strategy 'water production' produced similar priority maps to the consensus and 'keep clean' (aiming at clearing low density stands of invasive species) strategies in 
(a)
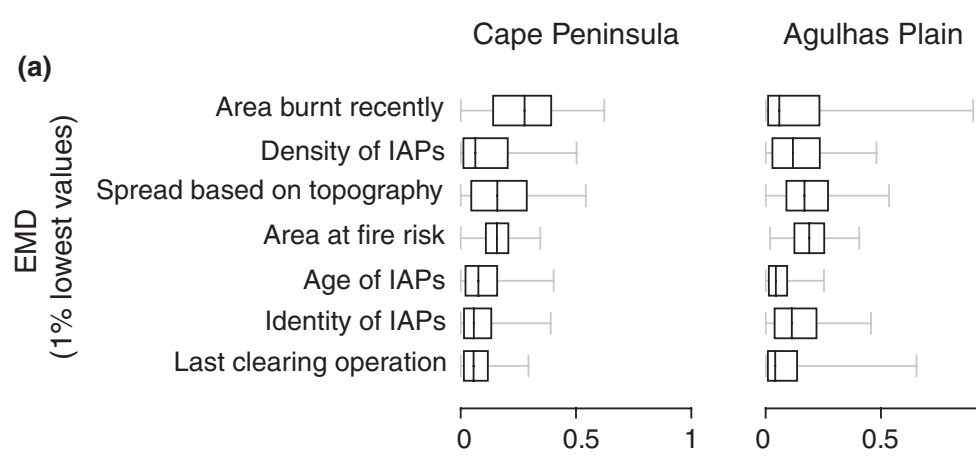

Outeniqua
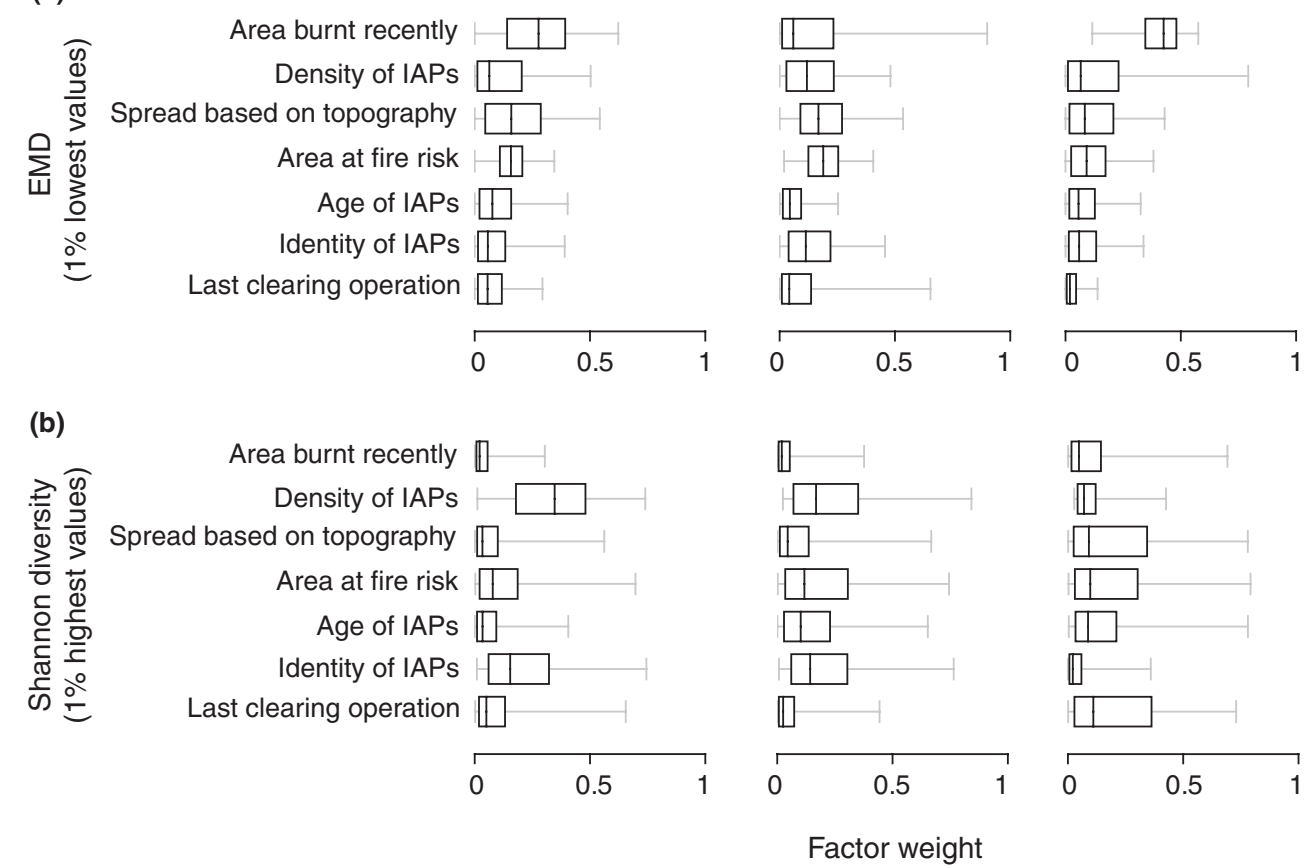

Figure 3 Box-plots showing the weights assigned to factors during the random weights analysis in three region in the Cape Floristic Region (Cape Peninsula, Agulhas Plain and Outeniqua) when considering: (a) the $1 \%$ subset of random weights closest to the consensus prioritization as measured the Earth Movers Distance (EMD), and (b) the $1 \%$ subset of random weights giving the highest diversity values as measured by the Shannon index. The analysis was performed by changing randomly the weights assigned to all factors included in the original decision model, in a way that the final weights sum up to 1 . The boxes correspond to the $25 \%$ and $75 \%$ percentile of all weights, the middle lines indicate the median value, and whiskers indicate the minimum and maximum weights. The factor weights of the original decision model appear indicated by an arrow. See the probability density of the weights assigned to factors in Fig. S6.

Cape Peninsula and Outeniqua; (2) in Agulhas Plain, the most similar priority maps occur between the consensus and 'maintain follow-ups' strategies; (3) the most different prioritization in Outeniqua is the one derived from the 'main follow-ups' strategy, while in Cape Peninsula and Agulhas Plain is only between the 'main follow-ups' and 'keep clean' strategies (Table 3). On the other hand, Pearson correlations suggested that: (1) the strategy 'water production' produced the most different priority maps in all three regions, and (2) Outeniqua is the region where major differences in the distribution of priorities were observed between strategies (Table 3). This latest observation is corroborated to some extent by the probability densities associate to each priority map shown in Fig. 4: Cape Peninsula presents a constant distribution of the priority values across all strategies, while Agulhas Plain and especially Outeniqua are more dependent on the adopted prioritization strategy.

\section{DISCUSSION}

\section{Sensitivity analysis framework: novelty and caveats}

This study presents a framework for a sensitivity analysis that evaluates the effects of changing the relative importance of factors (i.e. weights) included in a MCDM, by applying three different sensitivity approaches: one-dimensional, random and selected-weights analysis, which varied in the way the factor weights considered in the decision model were allowed to change (Butler et al., 1997). The first two approaches allowed us to evaluate the relative influence of individual factors and the linkages between the factors, whereas the third approach facilitated the comparison of the decision model under evaluation with other models of interest.

Besides the existence of multiple sensitivity analyses for MCDMs (Masuda, 1990; Triantaphyllou \& Sanchez, 1997; Hyde et al., 2005), our framework is novel in that it evaluates the influence of changes in factor weights in spatial dimensions. Instead of considering the outcomes of the model as discrete elements (i.e. cells in a given region that receive a priority value) and evaluating their rank order when changing the decision criteria (Butler et al., 1997; Fuller et al., 2008), changes in priorities are evaluated spatially explicitly using distance measures that take the spatial configuration into consideration. Each decision model is transformed into a spatial priority map, which is then compared to the original priority map using the EMD to identify spatial divergences between both prioritizations and the Shannon diversity index to measure the information contained in each model. The main limitation is, however, that the EMD gives us an idea of 
Table 2 Sets of factor weights derived from the random weights analysis producing: (a) the most similar priority maps to the consensus prioritization as measured by the Earth Movers Distance (EMD), and (b) the most informative priority maps as indicated by the Shannon diversity $(\mathrm{H})$ index, in three regions in the Cape Floristic Region (Cape Peninsula, Agulhas Plain and Outeniqua). The selection protocol followed Akaike Information Criterion (AIC) analysis, using EMD and $\mathrm{H}$ values to derive the likelihood function (see Methods for more details). When various sets of factor weights yielded the same minimum values of AIC, we selected those weight combinations that omitted the factors yielding a lower weight in the original decision model (they appear indicated by an asterisk $\left({ }^{*}\right)$ in the $n$-column). $\triangle \mathrm{AIC}$ refers to the difference in AICs between the consensus prioritization and the priority map derived from that specific set of weights; $\triangle \mathrm{AIC} \leq 2$ correspond to set of factor weights producing priorities for alien control similar to the consensus prioritization. We present the weights assigned to factors in the original decision model for comparison in the first row. See abbreviations in the Methods section.

\begin{tabular}{|c|c|c|c|c|c|c|c|c|c|c|c|c|}
\hline & $n$ & $\mathrm{ABR}$ & DoI & $\mathrm{SbT}$ & AFR & AoI & IoI & LCO & $\mathrm{EMD} / \mathrm{H}$ & $\log L i k$ & AIC & $\triangle \mathrm{AIC}$ \\
\hline Consensus & & 0.40 & 0.17 & 0.17 & 0.13 & 0.06 & 0.05 & 0.03 & & & & \\
\hline \multicolumn{13}{|l|}{ (a) } \\
\hline \multirow[t]{3}{*}{ Cape Peninsula } & 7 & 0.22 & 0.14 & 0.16 & 0.10 & 0.09 & 0.11 & 0.09 & 7.59 & -0.05 & 14.10 & -0.10 \\
\hline & 6 & 0.18 & 0.10 & 0.47 & - & 0.06 & 0.07 & 0.05 & 13.08 & -0.09 & 12.18 & 1.82 \\
\hline & 5 & 0.36 & 0.09 & - & - & 0.25 & 0.06 & 0.12 & 20.44 & -0.15 & 10.29 & 3.71 \\
\hline \multirow[t]{3}{*}{ Agulhas } & 7 & 0.07 & 0.23 & 0.27 & 0.17 & 0.08 & 0.09 & 0.07 & 9.84 & -0.02 & 14.05 & -0.05 \\
\hline & 6 & 0.08 & 0.19 & 0.26 & - & 0.14 & 0.07 & 0.09 & 21.92 & -0.05 & 12.11 & 1.89 \\
\hline & 5 & 0.49 & 0.10 & - & - & 0.07 & 0.14 & 0.10 & 32.93 & -0.08 & 10.16 & 3.84 \\
\hline \multirow[t]{3}{*}{ Outeniqua } & 7 & 0.46 & 0.12 & 0.08 & 0.09 & 0.06 & 0.06 & 0.13 & 11.45 & -0.07 & 14.13 & -0.13 \\
\hline & 6 & - & 0.46 & 0.09 & 0.07 & 0.13 & 0.06 & 0.12 & 30.17 & -0.18 & 12.36 & 1.64 \\
\hline & 5 & - & - & 0.15 & 0.16 & 0.27 & 0.07 & 0.24 & 33.41 & -0.20 & 10.41 & 3.59 \\
\hline \multicolumn{13}{|l|}{ (b) } \\
\hline \multirow[t]{3}{*}{ Cape Peninsula } & 7 & 0.09 & 0.26 & 0.13 & 0.14 & 0.21 & 0.07 & 0.06 & 4.20 & -0.01 & 14.02 & -0.02 \\
\hline & 6 & 0.20 & 0.27 & 0.11 & 0.26 & 0.07 & - & 0.06 & 4.23 & 0.00 & 12.01 & 1.99 \\
\hline & 5 & - & 0.43 & 0.23 & 0.16 & 0.07 & 0.08 & - & 4.24 & 0.00 & 10.01 & 3.99 \\
\hline \multirow[t]{3}{*}{ Agulhas } & 7 & 0.06 & 0.12 & 0.05 & 0.10 & 0.25 & 0.24 & 0.12 & 3.84 & 0.00 & 14.00 & 0.00 \\
\hline & 6 & - & 0.10 & 0.14 & 0.08 & 0.39 & 0.16 & 0.09 & 3.85 & 0.00 & 12.00 & 2.00 \\
\hline & 5 & - & 0.11 & 0.20 & 0.14 & 0.31 & - & 0.07 & 3.84 & 0.00 & 10.00 & 4.00 \\
\hline \multirow[t]{3}{*}{ Outeniqua } & 7 & 0.10 & 0.20 & 0.08 & 0.26 & 0.06 & 0.22 & 0.07 & 3.72 & 0.00 & 14.00 & 0.00 \\
\hline & $6^{*}$ & 0.11 & 0.18 & 0.46 & 0.06 & 0.09 & 0.06 & - & 3.72 & 0.00 & 12.00 & 2.00 \\
\hline & $5^{*}$ & 0.16 & 0.20 & 0.13 & 0.10 & 0.24 & - & - & 3.72 & 0.00 & 10.00 & 4.00 \\
\hline
\end{tabular}

the similitude between the two 'pictures' (or priority maps in our study case), but does not indicate the threshold above which the two maps can be considered sufficiently different. Likewise, the utility of the Shannon diversity index to describe the level of information contained in the priority maps and their effect on the management operations need further investigations. We only used these indices as relative distance metrics within a given region, but further developments would facilitate the refinement of the procedure and provide more clear/standard protocols on how to interpret the outputs of these analyses.

In this light, we also presented an incipient analysis for identifying strategies that provide similar outputs to the original consensus model by including less number of factors. Although highly promising in its objectives, the final results were complex and rather difficult to evaluate; changes in the original set of factor weights produced large changes in the outputs (i.e. priority maps) that precluded the achievement of reliable results (Drechsler, 2004). In this sense, considering the potentialities of the approach for guiding conservation practices and the scope for further methodological improvements, we encourage research to pursue this line and develop protocols for spatially-explicit sensitivity analysis applied to new management tools.

\section{Applications of sensitivity analysis in conservation: case study of biological invasions in the Cape Floristic Region}

As an example of the applicability of this sensitivity framework to evaluate the robustness of MCDMs, we applied the framework to a scheme previously developed for prioritizing areas for clearing of IAPs in any given management region within the Cape Floristic Region (Roura-Pascual et al., 2009). Results derived from the one-dimensional and random analyses indicate that major deviations from the original decision model in setting spatial-explicit priorities for IAPs control are produced when changing the weight assigned to the factor 'area burnt recently', but also when changing the weights assigned to 'density of IAPs' in Cape Peninsula and Agulhas Plain and to the 'last clearing operation' in Outeniqua. These results corroborate that consideration must be given to areas burnt recently when prioritizing areas for IAPs control. In all the studied regions, the factor 'area burnt recently' emerged as 


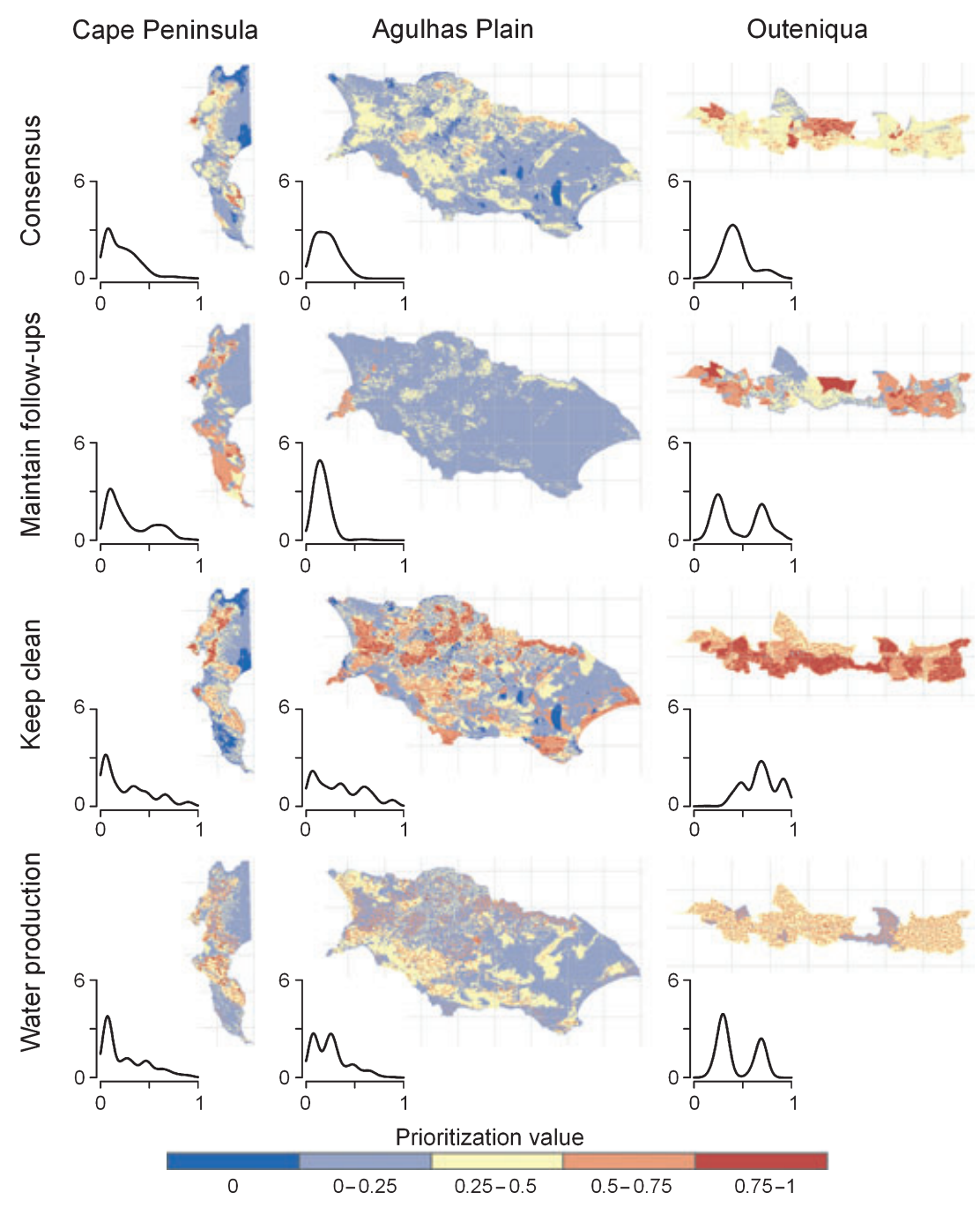

Figure 4 Priorities for management of invasive alien plants in three regions in the Cape Floristic Region (Cape Peninsula, Agulhas Plain and Outeniqua) following strategies considered in the selected-weights sensitivity analysis (Fig. 1). Colours correspond to priority values that range from low (0) to high (1) depending on the characteristics of the site and the weights assigned to factors. Graphs show to the distribution of the priority values (considering a bandwidth of 0.0005 ); $x$-axis refers to the priority value, and $y$-axis to the probability density. The size of the grid cells is $10 \times 10 \mathrm{~km}$.

the factor that produced the most marked changes in the spatial representation of priorities for IAPs control, and therefore accurate data for this factor are crucial. Managers need to be aware that incorporating wild fires or prescribed burning into their management options has important effects when prioritizing control operations. The importance of fire in controlling invasive species in the Cape Floristic Region has been highlighted in numerous studies (e.g. Kruger, 1983; Richardson et al., 1994; van Wilgen et al., 1994), and careful incorporation of this factor into management strategies is crucial. Although the relevance of the other two factors ('density of IAPs' and 'last clearing operations') depended on the geographic region, the divergences caused by these factors when included in the decision models need to be considered. The influence of 'density of IAPs' was expected considering that the different vegetation structures of the invaded stands have different management opportunities, but the influence of the 'latest clearing operation' in Outeniqua was somewhat surprising. We think that the relevance of 'last clearing operation' over 'density of IAPs' was a result of the characteristics of the study site: the density of invaded stands in Outeniqua is quite low thanks to the previous management operations, and therefore this factor does not seem to influence the final outputs of the model.

These divergences among regions indicate that the relative influence of factors included in the decision model is highly dependent on the characteristics of the study site and specifically on the spatial configuration of the environment. If the region under study is homogeneous in relation to one of the factors included in the analysis, this factor will have a low relevance even though it was assessed as highly important in the original decision model. This can be seen when looking at factor 'area burnt recently' in Agulhas Plain: the GIS layer underlying its spatial representation had a Shannon diversity of 
Table 3 Divergences in the distribution of the priorities for management of invasive alien plants in three regions in the Cape Floristic Region (Cape Peninsula, Agulhas Plain and Outeniqua) among strategies considered in the selected-weights sensitivity analysis (Fig. 1). The columns in the middle (from the second to the fifth) correspond to differences between strategies as measured by the Earth Movers Distance (EMD; below the diagonal) and Pearson correlations ( $r$; above the diagonal), while the last two columns indicate the degree of diversity and aggregation present in each prioritization strategy as measured by the Shannon diversity $(\mathrm{H})$ and evenness (E) indices, respectively.

\begin{tabular}{|c|c|c|c|c|c|c|}
\hline $\mathrm{EMD}^{r}$ & Consensus & Maintain follow-ups & Keep clean & Water production & $\mathrm{H}$ & $\mathrm{E}$ \\
\hline \multicolumn{7}{|l|}{ Cape Peninsula } \\
\hline Consensus & - & 0.61 & 0.82 & 0.41 & 4.43 & 0.71 \\
\hline Maintain follow-ups & 44.1 & - & 0.48 & 0.29 & 4.44 & 0.71 \\
\hline Keep clean & 33.1 & 63.9 & - & 0.38 & 3.82 & 0.67 \\
\hline Water production & 27.2 & 45.8 & 27.2 & - & 4.14 & 0.68 \\
\hline \multicolumn{7}{|l|}{ Agulhas Plain } \\
\hline Consensus & - & 0.80 & 0.91 & 0.32 & 4.07 & 0.73 \\
\hline Maintain follow-ups & 23.0 & - & 0.74 & 0.27 & 4.01 & 0.71 \\
\hline Keep clean & 36.3 & 41.7 & - & 0.26 & 4.11 & 0.74 \\
\hline Water production & 33.7 & 30.4 & 39.3 & - & 3.79 & 0.68 \\
\hline \multicolumn{7}{|l|}{ Outeniqua } \\
\hline Consensus & - & 0.48 & 0.33 & -0.04 & 4.07 & 0.86 \\
\hline Maintain follow-ups & 38.3 & - & 0.09 & 0.08 & 4.07 & 0.86 \\
\hline Keep clean & 32.9 & 35.8 & - & -0.30 & 3.10 & 0.82 \\
\hline Water production & 21.1 & 37.5 & 25.1 & - & 3.64 & 0.84 \\
\hline
\end{tabular}

0.01 (Fig. S2). Therefore, it can be expected that the weight for the factor 'area burnt recently' can be changed without major deviation from the consensus prioritization as corroborated by the systematic and random analyses. In contrast, changes in the weights assigned to factors with a low relevance in the original decision model might produce major changes in the final spatial representation of the priorities when the region is homogeneous in relation to the highly weighted factors and heterogeneous in relation to these low-weighted factors. These mismatches suggest that the decision model does not use the input data with maximum efficiency. What managers and researchers considered as important (i.e. high weight for the factor) when developing the decision model is not necessarily reflected in a high diversity of the underlying layer. This is obvious as the decision model was the same for any management region within the Cape Floristic Region, and layers depend on the temporal occurrence of the process determining them and also on the distinct environmental and socio-economic characteristics of the study site. The importance of the process under consideration does not necessarily translate into a spatially heterogeneous pattern.

In addition to evaluating the behaviour of the factors under different conditions, we also used the random analysis to identify the minimum number of factors that produce results similar to the consensus prioritization. This is important since data accessibility is a limitation in most cases, and the costs of collecting/preparing unnecessary data are not always acceptable. We used the classical model selection procedure based on the Akaike Information Criterion (AIC; Akaike, 1974), in which the EMD of each combination of factors and weights were used as the basis of the likelihood function. By applying this analysis to our study case, results indicate that the inclusion of most factors is necessary to produce similar results to the consensus prioritization. Even though some factors appeared to have a minor relevance on the final outputs, the removal of two or more factor from the decision model does not seem appropriate.

In contrast with the one-dimensional and random weights analyses, the selected-weighted sensitivity analysis permitted the search for divergences in the distribution of priorities for IAPs control among specific models. We compared the consensus prioritization derived from the decision-making process with experts on IAPs control with other management strategies currently under way in the Cape Floristic Region. The comparison showed that strategies provide different spatial distributions of the priorities, and this has considerable implications when allocating the resources available and targeting efficient actions for IAPs control. It is also expected that these differences are more accentuated in heterogeneous environments, since in homogeneous regions the effect of using one or another strategy is relatively indifferent because most of the areas will have similar priority values. These results indicate that the adoption of different prioritization approaches has considerable consequences at a spatial prioritization level and managers need to be aware of their decisions when setting priorities for alien control.

Our results show that sensitivity analysis is important for ensuring effective transfer of the state-of-the-art knowledge captured in sophisticated, spatially-explicit models to conservation management. First we identified the most influential factors in determining the establishment of priorities, and second the relevance of changes across geographic regions. The prioritization of areas for alien clearing is highly dependent on the regions of study in which it needs to be implemented. Even 
though some general guidelines can be derived, particular differences in environmental conditions and the status of the invasions preclude the generalization of the results across regions within the same bioregion. This has enormous management implications, since it shows the need to adjust prioritization strategies to the particularities of each region. However, we need to remember that this sensitivity approach only allowed us to examine divergences on the spatial prioritization among various strategies and the consensus prioritization in a static manner, but did not allow us to evaluate the dynamics of IAPs over time and therefore identify the optimal strategy (R.M. Krug et al., unpublished data). To provide final and more robust guidelines for the management of IAPs in South Africa's Cape Floristic Region, the development of a dynamic model considering the dispersal of the species under different management strategies needs to be conducted.

\section{CONCLUSIONS}

Considering the role of conservation biogeography in providing solutions to current human-nature linkages, and future changes associated with global environmental change, it is crucial to account for the level of uncertainty associated with our decisions (Fuller et al., 2008). Besides the substantial improvements in understanding the ecological and human mechanisms behind the dynamics of species distributions, there are still enormous uncertainties associated with the models used to synthesize the complexity of natural systems (Ascough et al., 2008). Although acknowledging these various uncertainties, few studies have explicitly included analysis of sensitivity of their decision models or future predictions. We find this lack of criticism a major drawback in analysing not only the problem at hand, but also in permitting further improvement on the field of conservation biogeography (Whittaker et al., 2005). We encourage research to investigate standard procedures to evaluate management practices and decision-making tools, and in particular the development of spatially-explicit sensitivity analyses as the one developed herein. Incorporating uncertainty into our analysis will allow us to have greater confidence in final outputs and managers will rely on research outputs to guide their decisions.

\section{ACKNOWLEDGEMENTS}

We thank the numerous people that helped us to understand the decision-making model and the many complexities involved in managing IAPs in the fynbos. We are especially grateful to Janet Franklin, Ingolf Kühn and an anonymous referee for their thoughtful and constructive comments on a previous version of the manuscript. The project was funded by the Global Environmental Facility (GEF) through the Cape Action for People and the Environment (CAPE) program. We also acknowledge financial support from the DST-NRF Centre of Excellence for Invasion Biology, the Catalan Agency for Management of University and Research Grants (Generalitat de Catalunya) through a Beatriu de Pinós Postdoctoral Grant (2006 BP-A 10124) to NR-P and a grant from the Hans Sigrist Foundation to DMR.

\section{REFERENCES}

Akaike, H. (1974) A new look at the statistical model identification. IEEE Transactions on Automatic Control, 19, 716-723.

Anderson, D.R. (2008) Model based inference the life science: a primer on evidence. Springer, Berlin.

Ascough, J.C., Maier, H.R., Ravalico, J.K. \& Strudley, M.W. (2008) Future research challenges for incorporation of uncertainty in environmental and ecological decision-making. Ecological Modelling, 219, 383-399.

Buckley, Y.M., Brockerhoff, E., Langer, L., Ledgard, N., North, H. \& Rees, M. (2005) Slowing down a pine invasion despite uncertainty in demography and dispersal. Journal of Applied Ecology, 42, 1020-1030.

Burnham, K.P. \& Anderson, D.R. (2002) Model selection and multimodal inference: a practical information-theoretic approach. Springer-Verlag, New York.

Butler, J., Jia, J.M. \& Dyer, J. (1997) Simulation techniques for the sensitivity analysis of multi-criteria decision models. European Journal of Operational Research, 103, 531-546.

Drechsler, M. (2004) Model-based conservation decision aiding in the presence of goal conflicts and uncertainty. Biodiversity and Conservation, 13, 141-164.

Fuller, M.M., Gross, L.J., Duke-Sylvester, S.M. \& Palmer, M. (2008) Testing the robustness of management decisions to uncertainty: everglades restoration scenarios. Ecological Applications, 18, 711-723.

Fürstenau, C., Badeck, F.W., Lasch, P., Lexer, M.J., Lindner, M., Mohr, P. \& Suckow, F. (2007) Multiple-use forest management in consideration of climate change and the interests of stakeholder groups. European Journal of Forest Research, 126, 225-239.

GRASS Development Team (2007) Geographic Resources Analysis Support System (GRASS GIS) Software). ITC-irst, Trento.

Harwood, J. \& Stokes, K. (2003) Coping with uncertainty in ecological advice: lessons from fisheries. Trends in Ecology \& Evolution, 18, 617-622.

Hurlbert, S.H. (1971) The nonconcept of species diversity: a critique and alternative parameters. Ecology, 52, 577586.

Hyde, K.M., Maier, H.R. \& Colby, C.B. (2005) A distancebased uncertainty analysis approach to multi-criteria decision analysis for water resource decision making. Journal of Environmental Management, 77, 278-290.

Krug, R.M. \& Eddelbuettel, D. (2009) Earthmovdist. R package version 0.1.0. Available at: http://r-forge.r-project. org/projects/earthmovdist.

Kruger, F.J. (1983) Plant community diversity and dynamics in relation to fire. Mediterranean-type ecosystems: the role of nutrients (ed. by F.J. Kruger, D.T. Mitchell and J.U.M. Jarvis), pp. 446-472, Springer-Verlag, Berlin. 
Levina, E. \& Bickel, P. (2001) The Earth Mover's distance is the Mallows distance: some insights from statistics. Proceedings Eighth IEEE International Conference on Computer Vision. ICCV 2001, 2, 251-256.

Ling, H. \& Okada, K. (2007) An efficient Earth Mover's Distance algorithm for robust histogram comparison. IEEE Transactions on Pattern Analysis and Machine Intelligence, 29, 840-853.

Masuda, T. (1990) Hierarchical sensitivity analysis of the priorities used in analytic hierarchy process. International Journal of Systems Science, 21, 415-427.

Moffett, A., Dyer, J.S. \& Sarkar, S. (2006) Integrating biodiversity representation with multiple criteria in North-Central Namibia using non-dominated alternatives and a modified analytic hierarchy process. Biological Conservation, 129, 181191.

Neubert, M.G. \& Caswell, H. (2000) Demography and dispersal: calculation and sensitivity analysis of invasion speed for structured populations. Ecology, 81, 16131628.

Peleg, S., Werman, M. \& Rom, H. (1989) A unified approach to the change of resolution: space and gray-level. IEEE Transactions on Pattern Analysis and Machine Intelligence, 11, 739-742.

R Development Core Team (2008) R: a language and environment for statistical computing). R Foundation for Statistical Computing, Vienna.

Regan, H.M., Davis, F.W., Andelman, S.J., Widyanata, A. \& Freese, M. (2007) Comprehensive criteria for biodiversity evaluation in conservation planning. Biodiversity and Conservation, 16, 2715-2728.

Richardson, D.M., van Wilgen, B.W., Le Maitre, D.C., Higgins, K.B. \& Forsyth, G.G. (1994) A computer-based system for fire management in the mountains of the Cape Province, South Africa. International Journal of Wildland Fire, 4, 17-32.

Roura-Pascual, N., Richardson, D.M., Krug, R.M., Brown, A., Chapman, R.A., Forsyth, G.G., Le Maitre, D.C., Robertson, M.P., Stafford, L., Van Wilgen, B.W., Wannenburgh, A. \& Wessels, N. (2009) Ecology and management of alien plant invasions in South African fynbos: accommodating key complexities in objective decision making. Biological Conservation, 142, 1595-1604.

Rubner, Y., Tomasi, C. \& Guibas, L.J. (2000) The earth mover's distance as a metric for image retrieval. International Journal of Computer Vision, 40, 99-121.

Saaty, T.L. (1980) The Analytic Hierarchy Process: planning, priority setting, resource allocation, McGraw-Hill, New York.

Shannon, C.E. (1948) A mathematical theory of communication. Bell System Technical Journal, 27, 379-423.

de Steiguer, J.E., Liberti, L., Schuler, A. \& Hansen, B. (2003) Multi-criteria decision models for forestry and natural resources management: an annotated bibliography. General Technical Report NE-307. United States Department of Agriculture, Forest Service, Northeastern Research Station, Newtown Square PA.
Triantaphyllou, E. \& Sanchez, A. (1997) A sensitivity analysis approach for some deterministic multi-criteria decisionmaking methods. Decision Sciences, 28, 151-194.

Valente, R.D.A. \& Vettorazzi, C.A. (2008) Definition of priority areas for forest conservation through the ordered weighted averaging method. Forest Ecology and Management, 256, 1408-1417.

Whittaker, R.J., Araujo, M.B., Paul, J., Ladle, R.J., Watson, J.E.M. \& Willis, K.J. (2005) Conservation biogeography: assessment and prospect. Diversity and Distributions, 11, 3-23.

van Wilgen, B.W., Richardson, D.M. \& Seydack, A.H.W. (1994) Managing fynbos for biodiversity: constraints and options in a fire-prone environment. South African Journal of Science, 90, 322-329.

Working for Water Program (2003) Standards for mapping and management of alien vegetation and operations data). Department of Water Affairs and Forestry, Cape Town.

\section{SUPPORTING INFORMATION}

Additional Supporting Information may be found in the online version of this article:

Figure S1 Scheme of the procedure used to convert any set of factor weights into a priority map.

Figure S2 Visualization of priorities for management of invasive alien plants at the factor level.

Figure S3 Visual interpretation of the effects of changing the priority maps on the Earth Movers Distance and the Shannon diversity indices.

Figure S4 Random weights sensitivity analysis based on the Earth Movers Distance and the Shannon diversity indices.

Figure S5 Frequency distributions of the Earth Movers Distance and the Shannon diversity indices derived from the random weight sensitivity analysis.

Figure S6 Probability density of the Earth Movers Distance and the Shannon diversity indices derived from the random weight analysis when considering $1 \%$ subset of sets of random weights.

Table S1 Source of the GIS data used to convert any set of factor weights into a priority map.

Table S2 Weights assigned to the different strategies considered in the selected-weights sensitivity analysis for setting spatiallyexplicit priorities for management of invasive alien plants.

Appendix S1 Description of the procedure used to convert any set of factor weights into a priority map.

As a service to our authors and readers, this journal provides supporting information supplied by the authors. Such materials are peer-reviewed and may be re-organized for online delivery, but are not copy-edited or typeset. Technical support issues arising from supporting information (other than missing files) should be addressed to the authors. 


\section{BIOSKETCH}

All coauthors are interested in the ecology of biological invasions and management opportunities for controlling their spread. The article originated from a Global Environmental Facility (GEF)-funded project conducted by the Centre for Invasion Biology (South Africa) to develop a dynamic-support model for prioritizing areas for invasive alien plant control in the Cape Floristic Region. Núria Roura-Pascual is currently a post-doctoral fellow at the Landscape Ecology and Biodiversity Lab (led by Lluís Brotons) at the Centre Tecnològic Forestal de Catalunya. Her particular interests include understanding the ecology of biological invasions in the context of global change and providing guidelines for their efficient management.

Author contributions: N.R.-P. and D.M.R. led the project and the writing of the article. R.M.K. and C.H. contributed to conceptual development, undertook analyses and assisted with the writing.

Editor: Janet Franklin 